\title{
Test Configuration for Incipient Fault Detection
}

\author{
Shuming Yang, Xiaoyu Wen, Xiaofei Zhang* \\ College of Basic Education, National University of Defense Technology, Changsha, 410073, China \\ ysmcsu@163.com \\ *zhangxiaofei@nudt.edu.cn
}

\begin{abstract}
Keywords: Equipment Health Management; Design for Testability; Test Optimization Configuration; Generic Algorithm
\end{abstract}

\begin{abstract}
Information sensing and test are premise and foundation of (Equipment Health Management, EHM), a reasonable sensor configuration not only provide accurate and complete fault information, but also improve fault diagnostics, fault prognostics and health state evaluation capability. To address the problem that the traditional test selection and optimization are mainly for fault detection and isolation, then, testability indices for EHM are firstly formulated quantitatively, then, test optimization selection model which minimizes test cost is modeled, and the generic algorithm is introduced to solve the problem. At last, a simulation case and an application case are given to verify \& validate the proposed model and method.
\end{abstract}

\section{Introduction}

As Equipment Health Management (EHM) technology getting mature, more and more complex equipment will take EHM into account in the design stage in the near future. At present, the existed researches are mainly focused on EHM architecture [1-2], PHM-related algorithms and models [3-5], few researches are given to information sensing and test of PHM. However, information sensing and test are premise and foundation of EHM [6-7]. Some applications and studies also show that PHM ability are more dependent on sensor information rather than on adopted models or algorithms [8], and the optimized test types, locations and numbers can enhance diagnostics, prognostics and health evaluation ability greatly [8].

Test optimization selection problem should be considered in the early design stage of equipment and be carried out concurrently with functional designing of weapon equipment. Design for Testability (DFT) embodies this ideal completely. Testability is a design characteristic which allows the status (operable, inoperable, or degraded) of an item to be determined and the isolation of faults within the item to be performed in a timely manner, Test Selection and Optimization (TSO) is one of the important contents in DFT.

Traditionally, the tests are primarily selected based on fault detection and isolation requirements rather than on EHM especially fault prognosis and health evaluation needs [9]. Against the backdrop, the paper mainly solve to problems: 1) how to describe the requirements of EHM for test quantitatively; 2) how to select the optimal tests for EHM.

\section{TSO Mode for EHM}

Fault diagnostics and prognostics are the key technologies to realize EHM, TSO should guarantee fault mode space to be fully detectable, isolatable and predictable. Based on the recognition, Universe Fault Detectable Rate (UFDR), Universe Fault Isolable Rate (UFIR), and Universe Fault Predictable Rate (UFPR) are proposed to describe the testability level for EHM.

Definition 1: UFDR is generally defined as, during the stated time span, the ratio of the number of faults detected correctly by sensors to the total number of equipment system faults.

Definition 2: UFIR is generally defined as, during the stated time span, the ratio of the number of faults isolated correctly to no more than the stated replaceable units by sensors to the number of the detected faults during the same time span. 
Definition 3: Possible Predictable Fault (PPF) is a progressive key fault or key components' fault, denoted by $F_{P P}$.

Definition 4: Predictable Fault (PF) is a PPF of which the early state is detectable, denoted by $F_{P}$.

Definition 4 describes whether a fault is predictable through the detectability of early state of the fault. Generally speaking, the early state detectability of a test depends on the main factors as follows:

(1) Signal to noise ratio (SNR): a high SNR implies fault detection uncertainty is small, while a low SNR implies that it is hard for the sensor to detect the fault.

(2) Timeliness (TIMN) is the ratio of the time span between the initiation of a fault (potential failure) and the detection of the fault by the sensor (time to detection, TTD) to the duration between the initiation of the fault and the time when the failure occurs (TTF).

(3) Sensitivity (SFDS) is the ratio of a sensor variation of per unit sensor resolution to a fault variation of per unit fault resolution.

Based on the three factors above, Test Fault Early State Detectability (TFESD) can be formulated by [8]:

$$
\operatorname{TFESD}_{i j}=\left\{\begin{array}{l}
\left(1+e^{-g\left(S N R_{j}-h\right)}\right)^{-1} *\left(1-\operatorname{TIMN}_{i j}\right)^{\alpha} *\left(1+e^{-b *\left(S E N S_{i j}-c\right)}\right)^{-1}, \text { if } T I M N_{i j}<1 \\
0, \text { if } \quad \operatorname{TIMN}_{i j} \geq 1
\end{array}\right\}
$$

Where TFESD $i j$ is the early state detectability of test $t_{j}$ for fault $f_{i}$, SNRj is the SNR of $t_{j}, g(=1)$ and $h(=0.5)$ are the shape parameters, $T I M N_{i j}$ is the timeless of $t_{j}$ for $f_{i}, \alpha(=0.5)$ is shape parameter, $S E N S_{i j}$ is the sensitivity of $t_{j}$ for $f_{i}, b(=10)$ and $c(=0.5)$ are shape parameters.

Definition 5: UFPR is defined as, during the stated time span, the ratio of the number of PF determined correctly by sensors to the total number of PPF of equipment system.

Given the fault set is $F=\left\{f_{1}, f_{2}, \ldots, f_{m}\right\}$, and the corresponding failure rate vector is $\lambda=\left[\lambda_{1}, \lambda_{2}, \ldots, \lambda_{m}\right]$. The complete test set used for selection is $T=\left\{t_{1}, t_{2}, \ldots, t_{n}\right\}$, and the corresponding sensor failure rate vector is $F R=\left[r_{1}, r_{2}, \ldots, r_{n}\right]$. A matrix $B=\left[b_{i j}\right]_{m \times n}$ is used to denote fault-sensor dependencies. The rows of $B$ correspond to faults, and the columns correspond to sensors. Element $b_{i j}$ is a two-tuple, $b_{i j}$ $=(u, v)$. And we suppose that if a sensor can detect the early state of a fault, it also means the sensor can track the fault evolution process. Then, if sensor $t_{j}$ can detect fault $f_{i}$ and its early state, then $b_{i j}$ $=(1,1)$. If sensor $t_{j}$ can detect fault $f_{i}$ but can not detect its early state, $b_{i j}=(1,0)$. If sensor $t_{j}$ can't detect fault $f_{i}$ nor its early state, then $b_{i j}=(0,0),\left(b_{i j}=0\right.$ for short $)$. Generally, if a sensor can detect early state of a fault, it also means that the sensor can detect the fault, so the case $b_{i j}=(0,1)$ will not exist.

Given $u$ denotes Boolean variable OR operation. And $\oplus$ denotes set XOR operation, when the two set are different, the operation result is true. $b_{i j}(k)$ denotes the $k$-th item of the two-tuple $b_{i j}=(u, v), k=1,2 . T_{f i}$ and $T_{f j}$ denote the sensor sets which can detect fault $f_{i}$ and fault $f_{j}$ respectively, i.e., $T_{f i}=\left\{t_{j} \mid b_{i j}(1)=1, t_{j}\right\}, T_{f i}$ is also called fault features of fault $f_{i} . F_{P P}$ denotes system PPFs. Given the ambiguity group size is $L$, then, the detectable faults set $F_{D}$, isolable faults set $F_{I}$ and predictable faults set $F_{P}$ are formulated respectively by:

$$
\left\{\begin{array}{l}
F_{D}=\left\{f_{i} \mid f_{i} \in F, \bigcup_{t_{j} \in T}^{n^{\prime}} b_{i j}(1)=1\right\} \\
F_{I}=\left\{f_{i} \mid f_{i} \in F_{D}, \sum_{f_{j} \in F} T_{f i} \oplus T_{f j} \leq L, \forall f_{j} \in F, f_{j} \neq f_{i}\right\} \\
F_{P}=\left\{f_{i} \mid f_{i} \in F_{P P} \cap F_{D}, \bigcup_{t_{j} \in T}^{n^{\prime}} b_{i j}(2)=1\right\}
\end{array}\right.
$$

According to Definitions 1, 2 and 5:

$$
U F D R=\sum_{f_{i} \in F_{D}} \lambda_{i} / \sum_{f_{i} \in F} \lambda_{i}, U F I R=\sum_{f_{i} \in F_{I}} \lambda_{i} / \sum_{f_{i} \in F_{D}} \lambda_{i}, U F P R=\sum_{f_{i} \in F_{P}} \lambda_{i} / \sum_{f_{i} \in F_{P P}} \lambda_{i}
$$

TSO model can be formulated by (4), which takes test cost as optimization objective, UFDR, UFIR and UFPR as constraint conditions. 


$$
\left\{\begin{array}{l}
T_{s}^{*}=\underset{T_{s}}{\arg \min } \sum_{t_{j} \in T_{s}} c_{j} \\
\text { s.t.UFDR } \geq U F D R^{*}, U F I R \geq U F I R^{*}, U F P R \geq U^{*}{ }^{*} R^{*}
\end{array}\right.
$$

Where $U F D R^{*}, U F I R^{*}$ and $U F P R^{*}$ are testability requirements that equipment system will satisfy.

Test Optimization Selection based on Generic Algorithm (GA):

Step1: Parameter initialization, including population size, PopSize, generic crossover and mutation probability, $p_{c}, p_{m}$, max iterative number, $N_{\max }$. The initialization population, Pop $=\left(x_{i j}\right)_{N \times n}$, is randomly generated, where $n$ denotes the number of sensors used for selection. When $t_{j}$ is selected, $x_{i j}=1$, otherwise, $x_{i j}=0$.

Step2: defining fitness function:

FitFun $=C_{0} /\left(\sum_{t_{j} \in T_{s}} c_{j}+\sum_{t_{j} \in T} c_{j}\right)-C_{1} \cdot \max \left(0, U F D R^{*}-U F D R\right)-C_{2} \cdot \max \left(0, U F I R^{*}-U F I R\right)-C_{3} \cdot \max \left(0, U F P R^{*}-U F P R\right)(5)$ Where $C_{0}, C_{1}, C_{2}$ and $C_{3}$ are constant.

Calculating individual fitness and justifying whether the iterative number satisfy the max iterative number. If true, outputting the optimal individual and the corresponding optimal solution, end; otherwise, going to step3.

Step3: Selecting individuals using roulette wheel selection method based on individual fitness, and executing crossover operation with probability $p_{c}$, hence producing population Pop'.

Step4: Executing mutation operation with probability $p_{m}$ on the individuals in population Pop', hence producing population $P$ op", return to step 2.

\section{Application analysis}

Diesel engine is a core system of some ship, and its operation state will have great impact on the mission success. In the design stage, the fault mode space of diesel engine should be sufficient observable in order to realize fault diagnostics, fault prognostics and health state evaluation in a quick, accurate and economic way. TBD234 diesel engine is of 9 subsystems, 44 modules, 100 faults and 76 tests [10]. Multi signal flow graph and TEAMS [11] are used to model the engine, shown in Figure 1. Further, the two-dimensional fault-test dependency matrix which is of $100 \times 76$ can be obtained from the model. Suppose that each test cost is $1, \mathrm{SNR}=10 \mathrm{~dB}, \mathrm{RESO}=1$.

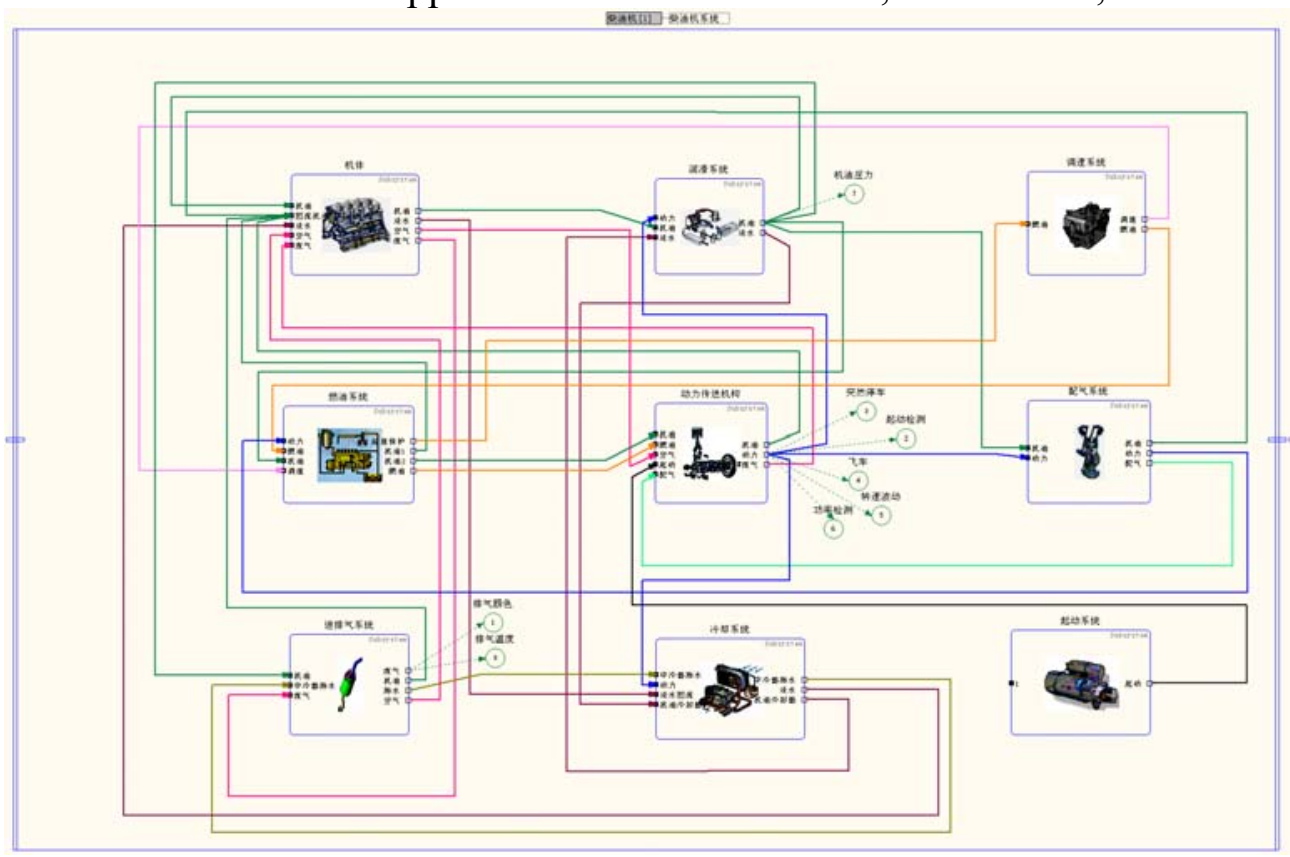

Fig.1. TBD234 diesel engine fault-test dependency model

According to EHM requirements of the TBD234, the required testability indices are: UFDR is 
not less than 0.95 , UFIR is not less than 0.90 and UFPR is not less than 1 GA parameters are set as: PopSize $=80, p_{c}=0.8, p_{m}=0.02, N_{\max }=100, C_{0}=10, C_{1}=C_{2}=C_{3}=0.5$.

The optimization tests are in Table 1 , accordingly, UFDR $=97.25 \%$, UFIR $=92.71 \%$, UFPR $=100 \%$, test $\cos t=32$.

Table 1 Test selection results for TBM234 EHM

\begin{tabular}{|c|c|c|c|}
\hline $\begin{array}{l}\text { Fuel cleaner obstruction } \\
\text { inspection }\end{array}$ & supercharger speed test & Fuel pressure & Water pipe inspection \\
\hline $\begin{array}{l}\text { Engine oil cleaner obstruction } \\
\text { inspection }\end{array}$ & Supercharger oil seal inspection & Engine oil pressure & $\begin{array}{ll}\begin{array}{l}\text { Liner water seal } \\
\text { detection }\end{array} & \\
\end{array}$ \\
\hline $\begin{array}{lll}\text { governor } & \text { buffer } & \text { spring } \\
\text { inspection } & & \\
\end{array}$ & Incubator inspection & Power detection & $\begin{array}{l}\text { Piston cylinder } \\
\text { jamming inspection }\end{array}$ \\
\hline $\begin{array}{l}\text { fuel injection pump plunger } \\
\text { spring inspection }\end{array}$ & Cool water temperature inspection & exhaust temperature & $\begin{array}{l}\text { Oil analysis in } \\
\text { governor }\end{array}$ \\
\hline inlet pressure test & $\begin{array}{l}\text { fuel supply advance } \\
\text { inspection }\end{array}$ & $\begin{array}{ll}\text { connecting } & \text { rod } \\
\text { mechanism } & \\
\end{array}$ & $\begin{array}{l}\text { heat exchanger pipe } \\
\text { obstruction detection }\end{array}$ \\
\hline $\begin{array}{lll}\text { cooling } & \text { pipe } & \text { obstruction } \\
\text { inspection } & & \end{array}$ & Start battery voltage & Fuel quality detection & $\begin{array}{l}\text { Oil analysis in } \\
\text { governor }\end{array}$ \\
\hline Inlet temperature test & Engine oil impurity detection & $\begin{array}{l}\text { pressure regulator valve } \\
\text { inspection }\end{array}$ & $\begin{array}{l}\text { Oil cooler fracture } \\
\text { detection }\end{array}$ \\
\hline Starting gear mesh & Starting switch test & Rotation rate inspection & $\begin{array}{l}\text { speed governor buffer } \\
\text { gear detection }\end{array}$ \\
\hline
\end{tabular}

\section{Conclusion}

EHM is of great significance to improve safety and availability of complex equipment, and good DFT can improve EHM capability. Based on the requirements of EHM for testability, the paper mainly presents TSO problem for EHM. The proposed model and method add fault predictable index into the existing fault detectable and fault isolatable indices, which enable the selected tests to satisfy the requirements of fault diagnostics and fault prognostics. Due to the NP-hard problem of the model, GA is introduced to solve the problem. At last, an equipment application validates the proposed method.

\section{Acknowledgement}

In this paper, the research was sponsored by the National Science Foundation of China (51405500), Pre-research Foundation (9140A17040114KG01242) and Science Foundation of National University of Defense Technology (ZK16-03-14 and JC14-09-01).

\section{References}

[1] Prosser W H, Allison S G, Woodard S E, et al. Structural health management for future aerospace vehicles[C]/Proc. of the 2nd Australasian Workshop on Structural Health Monitoring, $2004,8$.

[2] Discenzo F M, Nickerson W, Mitchell C E, et al. Open systems architecture enables health management for next generation system monitoring and maintenance[R]. The Office of Naval Research and the PEO Carriers Office Development Program White Paper,1999.

[3] Byington C S, Roemer M J, Galie T. Prognostic enhancements to diagnostic systems for improved condition-based maintenance[J].IEEE,2002, 2815-2824.

[4] Brotherton T. Prognosis of faults in gas turbine engines[J].IEEE,2000, 163-171.

[5] Roemer M J, Kacprzynski G J, Orsagh R F. Assessment of data and knowledge fusion strategies for prognostics and health management[J].IEEE,2001, 2979-2988.

[6] Keller K, Wiegand D, Swearingen K, et al. An architecture to implement integrated vehicle health management system[C]//.IEEE Systems Readiness Technology Conference.2001, 2-15. 
[7] L. Michael Santi, T.Shane Sowers, Robert B.Aguilar. Optimal sensor selection for health monitoring systems.NASA/TM-2005-213955.

[8] Guanfan Zhang. Optimum sensor localization/selection in a diagnostic/prognostic architecture [D]. Georgia Institute of Technology,2005.

[9] YANG Peng, QIU Jing, LIU Guan-jun, SHEN Qin-mu. The test selection algorithms based on Boolean logic[J].Journal of Test and Measurement technology.2007,21(5), pp. 386-390.

[10] ZHANG Shi-gang. Research on the optimization and generation techniques for diagnostic strategy based on multi-signal model[D]. National University of Defense Technology.2008, pp. $52-56$.

[11] Deb, S., Pattipati, K.R., Raghavan, V., Shakeri, M., and Shrestha, M., "Multi-Signal Flow Graphs: A novel Approach for System Testability Analysis and Fault Diagnosis," in Proc. IEEE AUTOTESTCON, Anaheim, CA, Sept. 1994. also IEEE AES Magazine, May 1995, pp. 14-25. 